\title{
Know your family ancestry, conditions, traits, and traditions (FACTs) DC: increasing genomic awareness in underserved communities
}

\begin{abstract}
Family Health History (FHH) is a practical, cost-effective, and engaging tool that is important in identifying individuals at an increased risk for disease. The "Know Your FACTs $\odot$ " (KYF) program has educated underserved communities about cancer risks, urged individuals to share their FHH with healthcare providers and family members, and encouraged cancer screening and risk reduction activities. In addition to containing instructions for the collection of FHH, the KYF tool included surveys which were distributed in underserved community-based settings to determine individuals' intentions to use and share their FHH. Our results demonstrate that $88.9 \%$ intend to use or utilized the FHH tool, $91.1 \%$ aim to share or shared the tool with their family members, $86.7 \%$ intend to share or shared their FHH with their doctor, and $53.3 \%$ would make lifestyle changes. Since the implementation of KYF, patient referrals to genetic counseling have increased considerably from 1 to 11 referrals monthly. These findings support the importance of the "Know Your FACTs $\odot$ " program by demonstrating that the community is interested in health and important services such as genetic counseling. As a result of this compelling effort, genetic counseling services are now being offered at the Howard University Cancer Center for the first time.
\end{abstract}

Keywords: genetics, community education, genomic education, family health history (FHH), underserved, african american, hispanic, public health, genetic counseling, screening tool
Volume 3 Issue 6 - 2015

\author{
Nicole Thompson, ${ }^{1,2}$ Altovise T Ewing, ${ }^{3}$ \\ JaVeatrice Bonner, ${ }^{1,2}$ Bradford Wilson, ${ }^{2}$ \\ Cherie Spencer,' Carla Williams,' Yasmine \\ M Kanaan, ${ }^{1,4}$ Amari Pearson-Fields, ${ }^{5}$ Luisel \\ Ricks-Santi I, 2,6 \\ 'Howard University Hospital, USA \\ ${ }^{2}$ Howard University National Human Genome Center, USA \\ 3Johns Hopkins University, USA \\ ${ }^{4}$ Howard University College of Medicine, USA \\ ${ }^{5}$ District of Columbia Department of Health, Community \\ Health Administration, Bureau of Cancer and Chronic Disease, \\ Cancer Programs Division, USA \\ ${ }^{6}$ Hampton University, USA
}

Correspondence: Luisel J Ricks-Santi, Director, Hampton University Cancer Research Center, 100 E. Queen Street, Hampton,VA 23668, USA, Tel 757-728-6034,

Email luisel.rickssanti@hamptonu.edu

Received: October 15, 2015 | Published: December 07, 2015
Abbreviations: AA, african american; BRCA, breast cancer gene; CGEN, cancer genetics education network; CME, continuing medical education; DC, district of columbia; FACTs, family ancestry conditions, traits, and traditions; FHH, family health history; GTC, genetic testing and counseling; $\mathrm{HBOC}$, hereditary breast and ovarian cancer; HUCC, howard university cancer center; KYF, know your facts; NHGC, national human genome center; PCP, primary care physician; USPTF, Us preventive services and task force

\section{Introduction}

The District of Columbia has the highest overall age-adjusted cancer mortality rate in the United States. ${ }^{1}$ Of special concern is our mortality ranking as 1 st in female colorectal $(18.8$ per 100,000$)$, prostate (37.6 per 100,000$)$, breast $(29.4$ per 100,000$)$, and cervical (3.7 per $100,000) .{ }^{2}$ Early detection and behavioral risk reduction activities can greatly reduce late stage diagnosis and cancer mortality. Remarkably, many cancers have strong familial and genetic components that can be identified to determine individuals at risk before diagnosis. ${ }^{3}$ Therefore, the collection of Family Health History (FHH) information may assist in early cancer detection; cancer risk reduction; improved utilization of cancer screening; chemoprevention; and surgical intervention for inherited cancers. We propose that incorporation and utilization of FHH in medical practice may also have an impact on the District's cancer mortality rate. FHH is a practical, cost-effective, and engaging tool that can be used to teach people how genetics, environment, and behaviors interact to affect health. It is well established that family health history is also a risk factor for many health problems including heart disease, asthma, cancer, diabetes, stroke, and even pregnancy related problems. ${ }^{4}$ By knowing one's FHH, individuals and families can make targeted lifestyle and screening choices to lower their risk of developing diseases that run in the family. In 2004, the U.S. Surgeon
General, Dr. Richard H. Carmona, launched a Family History Initiative where all Americans were encouraged to sit down with their families during the holiday season and collect their FHH. Thanksgiving Day was declared National Family History Day and has continued to be an annual event sponsored by the U.S. Surgeon General. ${ }^{5}$ A recent survey affirmed the public's growing interest in FHH. Results showed that 96\% of Americans felt knowing their FHH was important but only $30 \%$ collected information from their relatives to develop a $\mathrm{FHH}$. The lack of awareness of FHH by patients and their providers has far reaching health consequences, and diminishes opportunities for optimal health interventions.

\section{Purpose of the present study}

To address the burden of familial and genetically linked breast, colorectal, ovarian and prostate cancers in Washington, DC, first, the Howard University Cancer Center (HUCC), the National Human Genome Center (NHGC) at Howard University and the District of Columbia Department of Health developed and implemented an evidence-based community-level risk assessment tool aimed at collecting family medical history, for breast, colorectal, ovarian and prostate cancers. The Know Your Family Ancestry, Conditions, Traits, and Traditions (Know Your FACTs $\odot$, KYF) Initiative, is an outreach and educational program aimed at increasing awareness of the importance of obtaining family medical histories and the value of genetic testing and counseling. All materials and information were tailored to meet the cultural and linguistic needs of the African American and Latino community of Washington, DC. This project was a natural extension of the Community Genetics Education Network (CGEN) Project that aimed to increase awareness of genetic information and access to appropriate genetics education programs and services in underserved and underrepresented populations. The purpose of this study was to determine individuals' intentions to use 
and share their FHH once exposed to the Know Your FACTs $\odot$ FHH tool and to evaluate its impact on referrals for genetic counseling services. This study provides a descriptive analysis of the development and testing of our tool, as well as evaluation of impact on genetic counseling referrals.

\section{Methods}

\section{Participants}

Participants were African American and Hispanic individuals from various communities within the Washington, DC metro area between June and September 2012. Brochures were distributed to participants in locations that treat underserved communities such as Howard University Hospital and Howard University Hospital health fair and expositions, in addition to community partners working with underserved communities such as Nueva Vida (a support network for Latinas with cancer) and East of the River Clergy, Police, and Community Partnership. This study explored individuals' intentions to use and share their FHH once exposed to the KYF tool (Table $1 \&$ Table 2).

Table I Locations and dates of brochures' distribution and dissemination

\begin{tabular}{|c|c|c|}
\hline Distribution location & Number of brochures distributed & Date(s) \\
\hline Oncology Clinics & 90 & June - Sept. 2012 \\
\hline Health Fair (Community Health Expo) & 50 & |4-Jul-12 \\
\hline Dr. Oz Health Fair at Howard University & 100 & Sept. 5,2012 \\
\hline \multicolumn{2}{|c|}{ Howard University College of Medicine Department of Community and Family Medicine 50} & Sept. 13,2012 \\
\hline Howard University College of Medicine Department of Oncology & 50 & Sept. 13,2012 \\
\hline Howard University College of Medicine Department of Pediatrics & 50 & Sept. 13,2012 \\
\hline CGEN list serve & 250 & Sept. 14,2012 \\
\hline East of the River Clergy, Police, and Community Partnership & 50 & Sept. 14,2012 \\
\hline Howard University Cancer Center “Mammoday” Program & 40 & Sept. 15, 2012 \\
\hline Nueva Vida (community partner) & 75 & Sept. 15, 2012 \\
\hline Total & 805 & \\
\hline
\end{tabular}

Table 2 Characteristics of the study participants

\begin{tabular}{lll}
\hline Gender & Total number(45)\% & \\
\hline Male & 3 & $6.67 \%$ \\
Female & 42 & $93.33 \%$ \\
& & \\
Age & Total Number (45)\% & \\
I9-35 & 4 & $8.89 \%$ \\
$36+$ & 40 & $88.89 \%$ \\
Other & 1 & $2.22 \%$ \\
Education & Total Number (45)\% & \\
Some High school & 7 & $15.56 \%$ \\
High school diploma & 14 & $31.11 \%$ \\
Some College & 11 & $24.44 \%$ \\
College Degree & 10 & $22.22 \%$ \\
Graduate or Professional School & 3 & $6.67 \%$ \\
Race/Ethnicity & Total Number (44)\% & \\
African American & 26 & $59.09 \%$ \\
Caucasian & 4 & $9.09 \%$ \\
Latino/Hispanic & 8 & $18.18 \%$ \\
Asian & 1 & $2.27 \%$ \\
Biracial & 3 & $6.82 \%$ \\
Other & 2 & $4.55 \%$ \\
\hline
\end{tabular}

\section{Instrumentation}

The survey instrument consisted of items assessing demographic characteristics, including age, gender, zip code, education and selfreported racial/ethnic background. Participants were asked if they would utilize the tool themselves and if they would share it with family members or healthcare professionals. Additionally, participants provided information about changing lifestyle habits, such as smoking and exercise, as well as physicians' recommendations based on the information learned from the utilization of the KYF tool. Because several FHH instruments were available at the time of this study, KYF brochures were adapted from preexisting materials such as the Genetic Alliance's booklet on FHH "Does It Run In the Family?". The KYF materials included information about: 1) $\mathrm{FHH}, 2)$ genetic resources and services, and 3) cancer risk information. Specifically, the FHH information consisted of details on how and what information to collect from family members and a tool that could be used to facilitate the collection of one's FHH. Information about local resources for genetic 
counseling, education and testing available at the NHGC as well as the benefits of utilizing genetic testing and counseling for moderate to high risk African American and Latino/Latina residents were included. The document also provided risk information that included, but was not limited to, having 1) family members with cancer under the age of 50 and 2) many family members on the same side of the family with cancer. The tools were culturally and linguistically relevant for African Americans and Latino/Latina residents of Washington, DC and were also available free of charge.

\section{Procedures}

More than eight hundred and five brochures were distributed by the research team to African American and Hispanic residents, respectively, in community-based settings, at Howard University Clinics, cancer-screening clinics throughout the Washington, DC area, community health fairs, and directly to community partners (Table 1). Additionally, materials were mailed to HUCC community partners and individuals enrolled in the Howard University Consumer Genetics Education Network (CGEN) list serv. Completed surveys were collected and analyzed by the KYF team.

\section{Data analysis}

All brochures contained perforated surveys that could be mailed to the HUCC or handed in to community stakeholders. Postage was paid to increase survey participation. After receipt of surveys, descriptive statistics were performed.

\section{Results}

The KYF Team received forty-five surveys. The demographics of the individuals who completed the survey are listed in Table 2. Briefly, $93.3 \%$ of the surveys were completed by women, most of who were over the age of $36(88.89 \%)$. Education varied among the participants; $15.6 \%$ had some high school education while $31.1 \%$ received a high school diploma. More than $46.7 \%$ had some college or completed a college degree, whereas, $6.7 \%$ were graduated from professional schools. Regarding race and ethnicity, $59.1 \%$ of the respondents were African American while $18.8 \%$ were Latino or Hispanic. Of the respondents, $88.89 \%$ of the participants noted that they used or intend to use the FHH collection tool; $91.1 \%$ also checked that they intend to share the tool with their family members. Similarly, $77.78 \%$ checked that they would seek cancer screening after use of the tool. We did not inquire about reasons they would not seek cancer screening. Regarding their primary care doctors, $86.67 \%$ also revealed that they have or will share their FHH tool with their doctor. Most of the individuals indicated that they shared the tool with their physicians, and $42.22 \%$ of physicians made recommendations based on the patient's family history. After using the FHH tool, many participants noted that they would make behavioral changes. Specifically, $58.33 \%$ intend to do breast self-exams; $50 \%$ intend to change their diet; $70.83 \%$ intend to exercise; and $16.67 \%$ intend to quit smoking (Table 3) (Appendix).

Table 3 Survey results

\begin{tabular}{|c|c|c|c|c|}
\hline & Yes & $\%$ & Yes & $\%$ \\
\hline & \multicolumn{2}{|l|}{$N=(44)$} & \multicolumn{2}{|l|}{ n (Total Respondents) } \\
\hline Will you use this FHH tool? & 40 & $88.89 \%$ & $40(44)$ & $90.91 \%$ \\
\hline Will you share this FHH tool with your family? & 41 & $91.11 \%$ & $4 \mathrm{I}(44)$ & $93.18 \%$ \\
\hline Will you seek cancer screening after using this tool? & 35 & $77.78 \%$ & $35(42)$ & $83.33 \%$ \\
\hline Will you share this FHH tool with your doctor? & 39 & $86.67 \%$ & $39(43)$ & $90.70 \%$ \\
\hline $\begin{array}{l}\text { Did your doctor make any recommendations based on the information } \\
\text { from this FHH tool? }\end{array}$ & 19 & $42.22 \%$ & $19(33)$ & $57.58 \%$ \\
\hline $\begin{array}{l}\text { Did your doctor suggest you make any of the following lifestyle changes } \\
\text { after using this tool? }\end{array}$ & 24 & $53.33 \%$ & $14(24)$ & $58.33 \%$ \\
\hline \multicolumn{5}{|l|}{ Suggestions } \\
\hline Breast Self-Exam & & & $14(24)$ & $58.33 \%$ \\
\hline Diet Changes & & & $12(24)$ & $50.00 \%$ \\
\hline Exercise & & & $17(24)$ & $70.83 \%$ \\
\hline Quit Smoking & & & $4(24)$ & $16.67 \%$ \\
\hline
\end{tabular}

Table 4 Referral sources of participants referred to genetic counseling clinic

\begin{tabular}{lll}
\hline Referral sources & $\mathbf{n = 4 5}$ & Percentage\% \\
\hline Physician & 38 & $84.44 \%$ \\
Self-Referral & 5 & $11.11 \%$ \\
Referred by Patients' Navigator & 2 & $4.44 \%$
\end{tabular}

\section{Discussion}

A positive family history is a risk factor for many chronic diseases. Additionally, a positive family health history may also be an indication of a genetic predisposition, shared environments, and shared behaviors. By identifying women with a strong family history of breast cancer and providing genetic counseling and testing, we can provide patients with the tools necessary to reduce their risk of breast cancer - or prevent disease all together - in various ways. In some families, the development of some cancers, such as breast and ovarian, may be caused by a BRCA1 or BRCA 2 mutation and considered part of hereditary syndromes such as Hereditary Breast and Ovarian Cancer (HBOC) syndrome.6 In fact, the US Preventive Services Task Force (USPSTF)recommends that women with a family history of breast cancer are at increased risk for BRCA1 or BRCA2 mutations and should be referred for genetic testing and counseling (GTC). ${ }^{7}$ Conceivably, these women will benefit from genetic counseling - a resource that allows informed decision-making about testing and options to reduce their cancer risk. For example, women with BRCA1/2 genetic mutations have choices that include prophylactic surgery to prevent breast and ovarian cancer, chemoprevention, to surveillance or watchful waiting. There are limited data that support minority uptake of genetic counseling and testing. ${ }^{8-13}$ The majority of findings consistently reveal that US Whites access cancer genetic 
counseling and testing more frequently than AAs..$^{14}$ For example, Armstrong et al., found that women in a case-control study, seen in a university based primary care setting, African American women with a FHH similar to white women were less likely to undergo BRCA1 and BRCA2 genetic testing. ${ }^{15}$ Therefore, the use of GTC for primary cancer prevention is yet another area of medicine in which disparities occur. Family health history is an underutilized resource that has the potential to optimize individuals and familial health outcomes. A previous study found that when family medical histories are carefully and systematically obtained and collected, there is "moderately good agreement" between family histories obtained by family members and data obtained in the cancer registry. ${ }^{16}$ Therefore the collection of family medical history, particularly as it relates to breast, prostate, colorectal, and ovarian cancers, represents a valuable public health tool to assist individuals and their health care providers to accurately assess risk and develop care plans accordingly.

\section{Study limitations}

This study has limitations that may have restricted the effects our results. Over 800 brochures were distributed, however; only 45 surveys were received indicating a $5 \%$ return rate. This percentage does not reflect the utilization and effects of the KYF tool for the majority of individuals encountered. Participants might have found the information within the brochure valuable, but did not return their surveys for data collection. Additionally, the small sample size prevented us from performing some bivariate and multivariate analyses. Another limitation of the study is that our survey was assessed by self-report. Participants may have over- and/or underreported their behaviors regarding the impact of the KYF tool on their personal lifestyle, family health, and encounters with their healthcare providers. Also, several questions were written in past tense, and based on the assumption that the participant had time to utilize the FHH tool, speak with family members, and schedule an appointment with their healthcare physician to discuss the tool (if they chose to do so), prior to returning their survey to the researchers. The tool also provides a challenge for accurately identifying the participants who would benefit from a healthcare provider's recommendation to change lifestyle behaviors, such as smoking cessation or more frequent screenings. A few of the questions assume that the participant may have a significant personal and/or FHH, or lifestyle behaviors that would prompt the physician to suggest making a change. Although the number of surveys received did not represent the majority of the participants encountered, the FHH brochures increased awareness about FHH and encouraged community stakeholders to share their family health histories. This success is evident by the increase in referrals to genetic counseling.

\section{Research recommendations}

In the future, we plan to revise our survey tool to allow for more comprehensive reports from participants. We may also consider separating our survey into two parts, where the first will assess participant views and perceptions of the KYF brochure and information provided, while the second will assess the outcome of the tool regarding their utilization. Limited studies have been published evaluating the health care outcomes and decisions made between health care providers and patients equipped with their $\mathrm{FHH}$ information. Additional work will include a longitudinal analysis of outcomes associates with sharing $\mathrm{FHH}$ information with relatives. As a means of increasing physicians' abilities to incorporate $\mathrm{FHH}$ information into their practices, it would be helpful if providers could contribute and offer suggestions on the development of FHH tools that could be seamlessly integrated into their health care practices. These practices should be applied in an array of health care settings such as urban health care center, community health care center, outpatient clinics etc., in order to capture variations in use of FHH information in patient care. Future survey instruments should test the validation of the instrument and explore the impact of this instrument when made available online.

\section{Implications for genetic counseling practice}

As a result of our efforts, the genetic counseling clinic at the HUCC, has received many inquiries regarding genetic counseling and testing from community members and physicians. In total, 100 family health histories were collected by community members and evaluated by genetic counselors ( $\mathrm{n}=90$ at the HUH Oncology clinic and $n=10$ at the Community Health Expo). Notably, 38 (84.44\%) individuals were referred by their physicians, $5(11.11 \%)$ were self-referred, and $2(4.44 \%)$ were referred by community patient navigators (Table 4 ). Genetic counseling and testing has been an under-utilized resource within underserved communities, therefore having a program such as KYF available to provide this opportunity has been paramount to understanding the need for this service within the community. ${ }^{8,11,17}$ Additionally, having African-American and Latina genetic counselors, research assistants, and principal investigators as the leaders of this program has build trust within the community, in turn leading to successful outcomes and the opportunity to break barriers regarding minority and underserved recruitment and participation in research. African American genetic counselors may inherently have the skills to fill the gaps in effective communication that plague the health care system and contribute to health disparities, because of their understanding of the diverse cultures, backgrounds and issues that impact the community. Through KYF, underserved communities in the Washington DC metro area have learned of the availability of genetic testing, and as a result, the scientific community will learn of the genetic and genomic variations that are potentially playing a role in health outcomes within these communities. In fact, this activity resulted in the development of the cancer genetics counseling program at Howard University which recently found that AfricanAmerican and Hispanic women with breast cancer or with family histories of the disease likely will see a genetic counselor when given the opportunity to meet with one (manuscript in preparation). Also, the Howard University Cancer Center experienced a 40-fold increase in patients seeking genetic guidance with more than 200 patient encounters occurring within 21 months to two years after the implementation of the KYF program (manuscript in preparation). It is also well known that a barrier to utilization of genetic counseling and testing lies with the referring physician. The role of the physician in identifying and referring high-risk patients for genetic counseling cannot be underestimated. Advances in the field have necessitated that healthcare providers integrate genetics into the risk assessment and management of families with cancer. However, most primary care physicians (PCPs) have little experience or training relevant to genetic testing, and many lack knowledge and skill in this area of practice. ${ }^{18,19}$ Additionally, research has shown that minority-serving physicians were less likely to have ordered a genetic test to assess breast cancer risk and were also less likely to have referred a patient for genetic testing to a genetics center or counselor compared to those serving fewer minority patients. ${ }^{20}$ Moreover, Shields and colleagues found that physicians serving a disproportionate share of Medicaid patients were significantly less likely to have referred a patient to a genetic counselor, but those physicians who received training in clinical genetics though continuing medical education (CME) courses 
were significantly more likely to refer patients for genetic testing and counseling (GTC). In short, this is also critical issue that warrants intervention, especially in the age of precision medicine.

A study of 2,250 internists, gynecologists, and oncologists from the Eastern United States reported that knowledge of breast cancer genetics was relatively low overall, with only $25 \%$ of physicians answering all knowledge base questions in genetics correctly. ${ }^{21}$ Among oncologists, knowledge was associated with having a patient previously tested for BRCA mutations. Among primary care physicians, the numbers were even lower (40\%). Not surprisingly, physicians who felt confident and qualified to recommend counseling were much more likely to do so. Another important factor in predicting genetic services is patient inquiry as to whether they can or should be tested. ${ }^{22}$ Even if a physician fails to recommend genetic counseling, informed individuals may act as their own health care advocates. This data supports training and education for both PCP and patients, which we will address in the next phase of the KYF program.

\section{Conclusion}

Obtaining an accurate family medical history is particularly important in identifying individuals who may be predisposed to various cancers. Family health history has also been a valuable tool in preventive medicine. Family history taking, as part of a diseasespecific approach, aims to identify the risk of selected single gene disorders for the purpose of offering appropriate specialist referral and management. However, family histories must be adequate to enable PCPs to make appropriate clinical and prevention decisions. Ultimately, we believe that the success of this project was due to facilitating accurate FHH collection at the community level. We show that the community is interested in their health and that our efforts to increase awareness of $\mathrm{FHH}$ also increased the use of genetic counseling services at Howard University. More specifically, AA have reported that cancer genetic risk assessment would help: a) motivate them to perform breast self-examinations and/or mammograms more regularly; b) inform family members about their potential risk of developing cancer; and c) improve decision-making regarding preventive care. These findings supported the development and implementation of our "Know Your FACTs" program and educational materials in communities of color like Washington, DC where the population is one of the most diverse in the nation.

\section{Acknowledgements}

None.

\section{Conflicts of interest}

The authors declare that there is no conflict of interest.

\section{References}

1. Price RA, Blanchard JC, Harris R, et al. Monitoring cancer outcomes across the continuum: Data Synthesis and Analysis for the District of Columbia. Rand Health.

2. Siegel RL, Miller KD, Jemal A. Cancer Statistics 2015. CA A Cancer J Clin. 2015;65(1):5-29.

3. Schrader KA, Sharaf R, Alanee S, et al. Genetic Factors: Hereditary Cancer Predisposition Syndromes. In: Niederhuber JE, editor. Abeloff's Clinical Oncology. 5th edn. Philadelphia, USA. Elsevier; 2014:169-187.

4. Yoon PW, Scheuner MT, Jorgensen C, et al. Developing family healthware, a family history screening tool to prevent common chronic diseases. Prev Chronic Dis. 2009;6(1):A33.
5. NIH. The US surgeon general's family history initiative: My family health portrait. National Human Genome Research Institute (NHGRI). 2014.

6. Antoniou A, Pharoah PD, Narod S, et al. Average risks of breast and ovarian cancer associated with BRCA1 or BRCA2 mutations detected in case series unselected for family history: A combined analysis of 22 studies. Am J Hum Genet. 2003;72(5):1117-1130.

7. US Preventive Services Task Force. Genetic risk assessment and BRCA mutation testing for breast and ovarian cancer susceptibility: Recommendation statement. Ann Intern Med. 2005;143(5):355-361.

8. Glanz K, Grove J, Lerman C, et al. Correlates of intentions to obtain genetic counseling and colorectal cancer gene testing among at-risk relatives from three ethnic groups. Cancer Epidemiol Biomarkers Prev. 1999;8(4Pt2):329-336.

9. Halbert $\mathrm{CH}$, Kessler L, Troxel AB, et al. Effect of genetic counseling and testing for BRCA1 and BRCA2 mutations in african american women: A randomized trial. Public Health Genomics. 13(7-8):440-448.

10. Halbert $\mathrm{CH}$, Kessler L, Collier A, et al. Low rates of african american participation in genetic counseling and testing for $\mathrm{BRCA} 1 / 2$ mutations: Racial disparities or just a difference? J Genet Couns. 2012;21(5):676683

11. Lerman $\mathrm{C}$, Hughes $\mathrm{C}$, Benkendorf $\mathrm{JL}$, et al. Racial differences in testing motivation and psychological distress following pretest education for BRCA1 gene testing. Cancer Epidemiol Biomarkers Prev. 1999;8(4Pt2):361-367.

12. Sheppard VB, Mays D, LaVeist T, et al. Medical mistrust influences black women's level of engagement in BRCA 1/2 genetic counseling and testing. $J$ Natl Med Assoc. 2013;105(1):17-22.

13. Sheppard VB, Graves KD, Christopher J, et al. African american women's limited knowledge and experiences with genetic counseling for hereditary breast cancer. J Genet Couns. 2014;23(3):311-322.

14. Gramling R, Clarke J, Simmons E. Racial distribution of patient population and family physician endorsed importance of screening patients for inherited predisposition to cancer. $J$ Health Care Poor Underserved. 2009;20(1):50-54.

15. Armstrong K, Micco E, Carney A, et al. Racial differences in the use of BRCA $1 / 2$ testing among women with a family history of breast or ovarian cancer. JAMA. 2005;293(14):1729-1736.

16. Kerber RA, Slattery ML. Comparison of self-reported and databaselinked family history of cancer data in a case-control study. Am J Epidemiol. 1997;146(3):244-248.

17. Simon MS, Petrucelli N. Hereditary breast and ovarian cancer syndrome : The impact of race on uptake of genetic counseling and testing. Methods Mol Biol. 2009;471:487-500.

18. Escher M, Sappino AP. Primary care physicians' knowledge and attitudes towards genetic testing for breast-ovarian cancer predisposition. Ann Oncol. 2000;11(9):1131-1135.

19. Pichert G, Dietrich D, Moosmann P, et al. Swiss primary care physicians' knowledge, attitudes and perception towards genetic testing for hereditary breast cancer. Familial cancer. 2003;2(3-4):153-158.

20. Shields AE, Burke W, Levy DE. Differential use of available genetic tests among primary care physicians in the united states: Results of a national survey. Genet Med. 2008;10(6):404-414.

21. Doksum T, Bernhardt BA, Holtzman NA. Does knowledge about the genetics of breast cancer differs between nongeneticist physicians who do or do not discuss or order BRCA testing? Genet Med. 2003;5(2):99105

22. Wideroff L, Vadaparampil ST, Breen N, et al. Awareness of genetic testing for increased cancer risk in the year 2000 national health interview survey. Community Genet. 2003;6(3):147-156. 